\title{
Beyond Bonferroni Revisited: Concerns over inflated false positives in conservation genetics, genetics, and neuroscience
}

\author{
Tonya White, $\mathrm{MD}, \mathrm{PhD}^{1,2}$ \\ Jan van der Ende ${ }^{1}$ \\ Thomas E. Nichols, $\mathrm{PhD}^{3,4,5}$ \\ ${ }^{1}$ Department of Child and Adolescent Psychiatry \\ ${ }^{2}$ Department of Radiology \\ Erasmus University Medical Center, Rotterdam, the Netherlands \\ Neurosciences, University of Oxford, Oxford, OX3 9DU \\ ${ }^{5}$ Department of Statistics, University of Warwick, Coventry, CV4 7AL, UK
}

${ }^{3}$ Oxford Big Data Institute, Li Ka Shing Centre for Health Information and Discovery, Nuffield Department of Population Health, University of Oxford, Oxford, OX3 7LF, UK

${ }^{4}$ Wellcome Centre for Integrative Neuroimaging, FMRIB, Nuffield Department of Clinical

\section{Corresponding Author}

Tonya White, M.D., Ph.D., M.Sc. Eng

Associate Professor

Department of Child and Adolescent Psychiatry

Erasmus MC-Sophia / Kamer KP-2869

Postbus 2060

3000 CB Rotterdam

tel: $+31(0) 10703.70 .72$

Email: t.white@erasmusmc.nl

Keywords: Multiple Testing Correction, False Discovery Rate, Family Wise Error, Benjamini Hochberg, Benjamini Yekutieli 


\begin{abstract}
In 2006, Narum published a paper in Conservation Genetics that was motivated by the stringent nature of the Bonferroni approach for family wise error correction. That work suggested that the approach of Benjamini and Yekutieli in 2001 provided adequate correction and was more biologically relevant. However, there are crucial differences between the original Benjamini and Yekutieli procedure and that described by Narum. After carefully reviewing both papers, we believe that the Narum procedure is both different than the Benjamini and Yekutieli procedure and does not adequately control for family wise error. We provide an overview of approaches for FWE correction as well as evidence for the faulty implementation of the Benjamini and Yekutieli procedure by Narum using the equations from the respective papers, data from both papers, and the results of simulation.
\end{abstract}




\section{Introduction}

In 2006, Narum published a paper in Conservation Genetics motivated by the stringent nature of the Bonferroni approach for multiple testing correction, suggesting the False Discovery Rate (FDR) method proposed by (Benjamini and Yekutieli 2001) as an alternative that is both powerful but also more biologically relevant. His paper titled "Beyond Bonferroni: Less conservative analyses for Conservation Genetics" has been cited over 500 times [https://link.springer.com/article/10.1007/s10592-005-9056-y]. The article has not only been cited in the field of conservation genetics, but also has been increasingly cited in the fields of medicine and neuroscience. These studies apply the approach of Narum (2006) attributed to the Benjamini and Yekutieli (2001) (BY) procedure for muliple testing correction.

However, a careful review of the published BY approach and what Narum describes as the BY method, there are crucial differences. Due to an omission of one term, Narum's implementation of BY is incorrect and cannot be guaranteed to control the FDR. Thus, we believe that the Narum publication has created confusion about the BY procedure and its misuse is being propogated along an increasing number of studies. Thus, we have two goals of this paper: The first is to provide an overview of the Bonferroni method, the original Benjamini \& Hochberg (2000) FDR (BH-FDR), and BY's method (BY-FDR); the second goal is to describe faulty implimentation of the BY-FDR approach described by Narum. We will demonstrate that using the multiple testing correction described by Narum results in an excessive number of false positives, especially when a larger number of multiple tests are performed.

\section{Theory}


We first review the different multiple testing approaches discussed by Narum (2006) using his notation as closely as possible. For a collection of $k$ tests, each with a corresponding pvalue, $p_{i}, i=1, \ldots, k$. A multiple testing procedure identifies a subset of the $k$ tests as significant while controlling for some measure of false positive risk that takes into account the number of tests performed. The Bonferroni method controls the family-wise error (FWE), the chance of one or more false positives, by using a fixed threshold of:

$$
\alpha_{\mathrm{Bonf}}=\frac{1}{k} \alpha_{\mathrm{FWE}}
$$

where $\alpha_{\mathrm{FWE}}$ is the desired FWE level: All tests with $p_{i} \leq \alpha_{\mathrm{Bonf}}$ can be declared significant while controlling the FWE.

Benjamini \& Hochberg (2000) introduced the False Discovery Rate (FDR) for multiple testing correction. In describing the FDR it is useful to first define the false discovery proportion (FDP): FDP is the ratio of the number of false positive tests to total number of significant tests, defined as 0 if no tests are significant. The FDR is the expected value of FDP; put another way, FDR is the expected proportion of false positives among positives. To find FDR-significant tests, denote the ordered p-values $p_{(1)} \leq p_{(2)} \leq \cdots \leq p_{(k)}$. Then for a desired $\alpha_{\mathrm{FDR}}$, let the index $i^{*}$ be found as

$$
i^{*}=\max \left\{i: p_{(i)} \leq \frac{1}{k} \alpha_{\mathrm{FDR}}\right\}
$$

and the tests with $p_{i} \leq p_{\left(i^{*}\right)}$ can be declared significant while controlling FDR at $\alpha_{\mathrm{FDR}}$.

The assumptions of this Benjamini \& Hochberg FDR procedure (BH-FDR) are independence among the test statistics (Benjamini \& Hochberg, 2000). However, BY found that weaker assumptions could be used, allowing a general form of positive dependence among the test statistics. The BY work, however, also proposed another method for controlling FDR that makes no assumptions about the dependence among the tests, as long as a more stringent criterion was used (Theorem 1.3, BY), with the index $i_{\mathrm{BY}}{ }_{\mathrm{BY}}$ computed: 


$$
i_{\mathrm{BY}}^{*}=\max \left\{i: p_{(i)} \leq \frac{1}{k} \frac{1}{\sum_{i^{\prime}=1 i^{\prime}}^{k}} \alpha_{\mathrm{FDR}}\right\}
$$

With this approach, the tests with $p_{i} \leq p_{\left(i_{\mathrm{BY}}^{*}\right)}$ are marked significant and FDR is controlled at $\alpha_{\mathrm{FDR}}$ under any form of dependency. Notably $\sum_{i^{\prime}=1}^{k} \frac{1}{i^{\prime}} \approx \log (k)+\gamma$, where $\gamma \approx 0.57721$ is Euler's constant. This is the method we refer to by BY-FDR.

We can now make a quick comparison of three methods on the basis of the smallest p-value $p_{(1)}$ : Bonferroni has the fixed threshold $\alpha_{\mathrm{FWE}} / k$, while BH-FDR will compare $p_{(1)}$ to $\alpha_{\mathrm{FDR}} / k$ and BY-FDR will compare $p_{(1)}$ to approximately $\alpha_{\mathrm{FDR}} /(k \log (k))$. Of course, BH-FDR and BY-FDR are adaptive and compare increasing p-values to successively more lenient thresholds, but this comparison for $p_{(1)}$ points to how BY-FDR is much more stringent than BH-FDR.

Now, in Narum (2006), the author incorrectly states that the BY-FDR threshold is fixed and equal to:

$$
\frac{1}{\sum_{i=1}^{k} \frac{1}{i}} \alpha_{\mathrm{FDR}}
$$

This is a fundamental error, as a key feature of FDR methods is that they are adaptive. The error arose from neglecting that the equation above was merely one component of the BY procedure (to be substituted for q in B-Y Eq. (1) on pp. 1167 (Benjamini and Yekutieli 2001)). The Narum procedure results in a fixed threshold for a specific $k$.

Since a fixed threshold specifies the average or per comparison error rate (PCE), we can assess the impact of this error. Assuming the complete null, i.e. no signal for any test, $k \times$ PCE is the expected number of false positives. For the threshold at the 0.05 level, for $k=105$, $k \times \mathrm{PCE} \approx 1$, while for $k=1590, k \times \mathrm{PCE} \approx 10$. This demonstrates that Narum's result can be assured to produce an increasing number of false positives for an increasing $k$. In contrast, for Bonferroni $k \times$ PCE is exactly $\alpha_{\mathrm{FWE}}$, i.e. always less than 1 , and every valid FWE or FDR 
level $\alpha$ procedure is guaranteed to produce no false positives with probability $1-\alpha$ (again, in this complete null setting). While the Narum approach does asymptote to zero as $k$ approaches infinity, it approaches zero extremely slowly. For example, with 10 million tests performed, the Narum p-value threshold is 0.003 , in contrast to the Bonferroni threshold of 0.000000005 .

To evaluate the rate of significant p-values between the Bonferroni, B-H, B-Y, and Narum's interpretation of the B-Y approach we conducted a simulation. We created 50,000 random realizations where random p-values were computed from a standard Normal distribution. We considered $k$ ranging from 1 to 30 tests, where all tests were independent, and used nominal $\alpha_{\mathrm{FWE}}=\alpha_{\mathrm{FDR}}=0.05$ for all methods. In this null setting, any "discovery" is a false discovery and so measured FDR and FWE will be the same. So we computed the proportion of realizations where any p-values we found significant (FDR \& FWE), as well as the proportion of tests among the $\mathrm{k}$ that were false positives, known as the Per Comparison Error rate (PCE). Python code is available in Appendix 1.

Figure 1 shows the FDR and FWE as a function of the number of tests, showing that Bonferroni and BH-FDR both control false positives as expected (as an aside, while Bonferroni is often regarded as conservative, in this setting of small $k$ and independent tests, it is essentially exact). The FDR/FWE of BY-FDR becomes increasing conservative while Narum's method has inflated false positives even for $\mathrm{k}=2$ tests, and has a near linear increase with increasing $k$. In all realizations there was never more than 1 detection, and hence the PCE was identical to the FDR/FWE (not shown).

We also consider the specific set of 15 p-values used in Narum (2006), tabulating the pvalue threshold that were used for significance testing for each of the four methods. Table 1 shows the thresholds used for each of the 15-exemplar p-values, with significant tests marked in bold. It can be seen that the BY-FDR and the Narum approach are not the same, with Narum finding 4 significant tests as compared to BY-FDR's having two significant tests. 


\section{Discussion}

Approaches for multiple testing correction have been present for over half a century. In the late 1950's, Olive Jean Dunn adapted the Italian mathematician Carlo Emilio Bonferroni's theory of inequalities for use in statistics (Dunn 1961). However, since the Bonferroni approach is one of the most conservative, especially when the multiple tests are dependent, multiple alternative approaches to correct for multiple testing have surfaced. Two commonly used approaches include the Benjamini \& Hochberg that was introduced in 1995 and the Benjamini and Yekutieli approach that was introduced in 2001. In 2006, Narum published a paper which provided an overview and examples of the BY-FDR procedure. However, we demonstrate that the approach described by Narum is an incorrect depiction of the BY-FDR approach.

We believe that Narum used an equation from the BY paper (shown above) out of context. A careful reading of Benjamini and Yekutieli (2001) finds that this expression (from Theorem 1.3 on pp. 1169 of BY) is to be entered as the $\alpha$ in the B-H equation (Eq. (1) on pp. 1167 in BY), producing an adaptive threshold. Further, we also show, based on a series of p-values taken also from the Narum paper (Table 1) that different results are obtained from the BYFDR approach and the Narum approach.

Direct calculation shows that Narum's incorrect implementation of BY-FDR has expected number of false positives that increases linearly with number of tests $k$, and invalid and increasing false positive rates that, crucially, are drastically different from BY-FDR's valid but conservative performance. We believe that over 500 publications citing Narum (2006) are liable to have this inflated rate of false positives in their results. For example, we are particularly concerned about papers that cite both the BY paper as the method used, but use the multiple testing correction described by Narum 2006 (Chye et al.; Whittle et al. 2016; Woolley et al. 2018). 
We do agree with Narum that the Bonferroni approach is often conservative for multiple testing correction, especially with dependent data. However, there has also been a growing concern that many studies fail to replicate (Ioannidis 2005; Open Science Collaboration 2015; Nichols et al. 2017). In the past, analyses were performed without adequately controlling for the numbers of tests performed (Carp 2012) which resulted in numerous type I errors. We know of no justification to use the procedure described by Narum for multiple testing, and are unaware of any formal metric of false positives that it controls. Thus, we would recommend that this approach not be used for multiple testing correction and the work corrected to properly impliment the BY-FDR approach. 


\section{References}

Benjamini Y, Hochberg Y (1995) Controlling the False Discovery Rate: A Practical and Powerful Approach to Multiple Testing. J R Stat Soc Ser B 57:289-300

Benjamini Y, Yekutieli D (2001) The Control of the False Discovery Rate in Multiple Testing under Dependency. Ann Stat 29:1165-1188. doi: 10.2307/2674075

Busard HLL (Hubertus LL (2010) Nicole Oresme, Questiones super geometriam Euclidis. Franz Steiner Verlag, Stuttgart

Carp J (2012) The secret lives of experiments: Methods reporting in the fMRI literature.

Neuroimage 63:289-300. doi: 10.1016/j.neuroimage.2012.07.004

Chye Y, Solowij N, Suo C, et al Orbitofrontal and caudate volumes in cannabis users: a multi-site mega-analysis comparing dependent versus non-dependent users. doi: $10.1007 / \mathrm{s} 00213-017-4606-9$

Dunn OJ (1961) Multiple comparison among means. J Am Stat Assoc 56:52-64

Eklund A, Nichols TE, Knutsson H (2016) Cluster failure: Why fMRI inferences for spatial extent have inflated false-positive rates. Proc Natl Acad Sci U S A 113:7900-5. doi: $10.1073 /$ pnas. 1602413113

Galwey NW (2009) A new measure of the effective number of tests, a practical tool for comparing families of non-independent significance tests. Genet Epidemiol 33:559-568. doi: $10.1002 /$ gepi.20408

Ioannidis JPA (2005) Why most published research findings are false. PLoS Med 2:06960701. doi: 10.1371/journal.pmed.0020124

Narum SR (2006) Beyond Bonferroni: less conservative analyses for conservation genetics. Conserv Genet 7:811-811. doi: 10.1007/s10592-006-9189-7

Nichols T, Das S, Evans AC, et al (2017) Best practices in data analysis and sharing in neuroimaging using MRI Best practices in data analysis and sharing in neuroimaging using MRI. Nat Neurosci 20:299-303. doi: 10.1038/nn.4500

Open Science Collaboration (2015) Estimating the reproducibility of psychological science. 
Science (80- ) 349:aac4716-aac4716. doi: 10.1126/science.aac4716

Whittle S, Liu K, Bastin C, et al (2016) Neurodevelopmental correlates of proneness to guilt and shame in adolescence and early adulthood. Dev Cogn Neurosci 19:51-57. doi: 10.1016/j.den.2016.02.001

Woolley JD, Chuang B, Fussell C, et al (2018) Intranasal oxytocin increases facial expressivity, but not ratings of trustworthiness, in patients with schizophrenia and healthy controls. doi: 10.1017/S0033291716003433 
Table 1 - A set of p-values from 15 significance testing taken from the Narum 2006 paper and comparison with four approaches to multiple testing. Numbers in bold reflect those in the p-value row that are significant.

\begin{tabular}{ccccc}
\hline \hline p-value & Bonferroni & Benjamini \& & Benjamini \& & Narum \\
Examples & & Hochberg & Yekutieli & \\
\hline \hline 0.0001 & $\mathbf{0 . 0 0 3 3}$ & $\mathbf{0 . 0 0 3 3}$ & $\mathbf{0 . 0 0 1 0}$ & $\mathbf{0 . 0 1 5 1}$ \\
0.0010 & $\mathbf{0 . 0 0 3 3}$ & $\mathbf{0 . 0 0 6 7}$ & $\mathbf{0 . 0 0 2 0}$ & $\mathbf{0 . 0 1 5 1}$ \\
0.0062 & 0.0033 & $\mathbf{0 . 0 1 0 0}$ & 0.0030 & $\mathbf{0 . 0 1 5 1}$ \\
0.0101 & 0.0033 & $\mathbf{0 . 0 1 3 3}$ & 0.0040 & $\mathbf{0 . 0 1 5 1}$ \\
0.0214 & 0.0033 & $\mathbf{0 . 0 1 6 7}$ & 0.0050 & 0.0151 \\
0.0227 & 0.0033 & $\mathbf{0 . 0 2 0 0}$ & 0.0060 & 0.0151 \\
0.0273 & 0.0033 & $\mathbf{0 . 2 3 3 3}$ & 0.0070 & 0.0151 \\
0.0292 & 0.0033 & $\mathbf{0 . 0 2 6 7}$ & 0.0080 & 0.0151 \\
0.0311 & 0.0033 & $\mathbf{0 . 0 3 0 0}$ & 0.0090 & 0.0151 \\
0.0323 & 0.0033 & $\mathbf{0 . 0 3 3 3}$ & 0.0100 & 0.0151 \\
0.0441 & 0.0033 & 0.0367 & 0.0111 & 0.0151 \\
0.0490 & 0.0033 & 0.0400 & 0.0121 & 0.0151 \\
0.0573 & 0.0033 & 0.0433 & 0.0131 & 0.0151 \\
0.1262 & 0.0033 & 0.0467 & 0.0141 & 0.0151 \\
0.5794 & 0.0033 & 0.0500 & 0.0151 & 0.0151 \\
\hline \hline
\end{tabular}


Figure 1 - Percentage of false positive results compared to the number of independent tests performed. The results were derived from computer simulations comparing four different approaches for multiple testing correction. Simulations were performed in python and utilized a 5000 iterations of a random Normal distribution converted to $\mathrm{p}$ values.

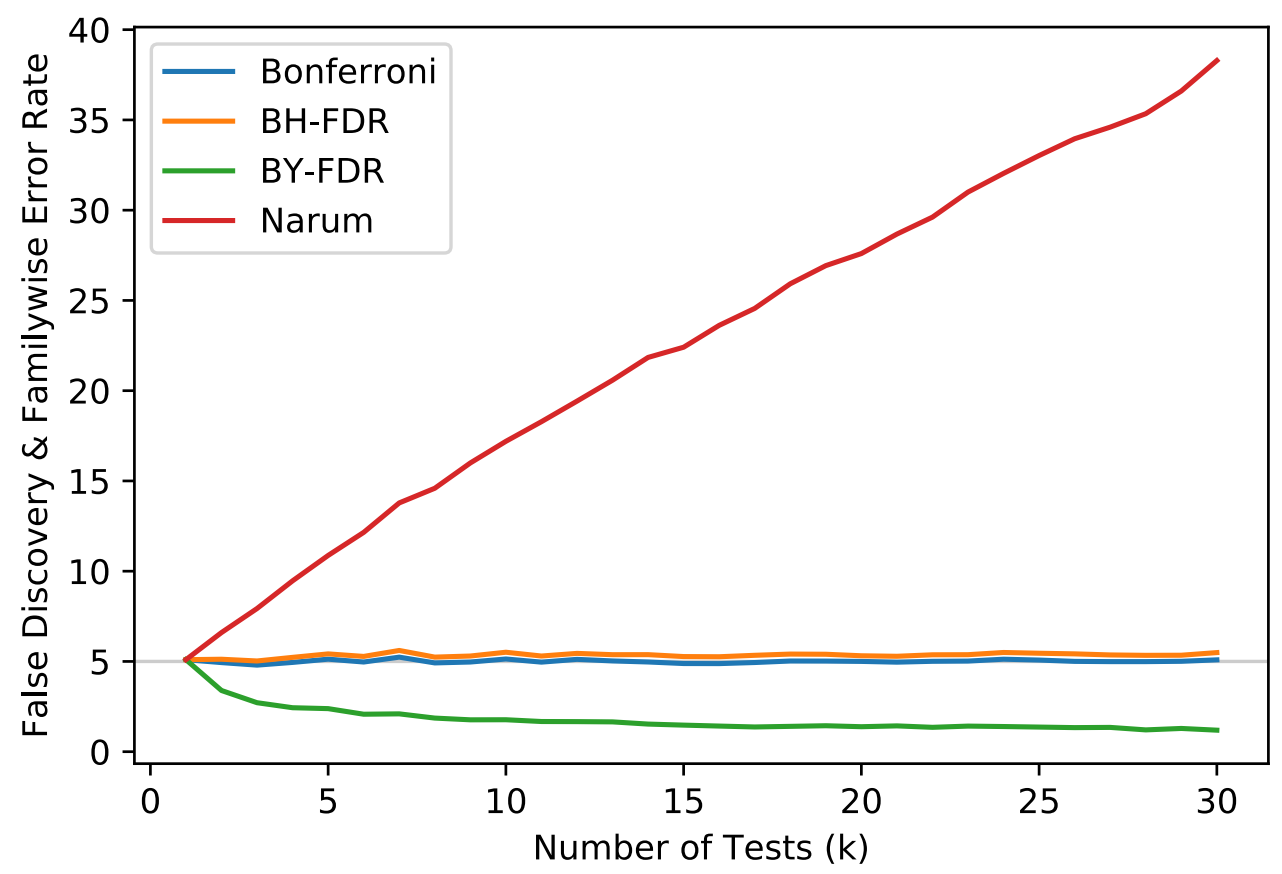




\section{Supplemental Material}

\section{Python Code for Simulation of the Multiple Testing}

"'""

Simulations performed on multiple testing in Python

Author: Tonya White, $\mathrm{MD}, \mathrm{PhD}$

Adapted by Tom Nichols, PhD on 3 September 2018

Date: 24 April 2018

Location: Rotterdam, Netherlands This program performs simulations of multiple testing to test the Narum paper
"'""

import numpy as np

import scipy.stats as st

import matplotlib as $\mathrm{ml}$

import matplotlib.pyplot as plt

from matplotlib import $\mathrm{cm}$

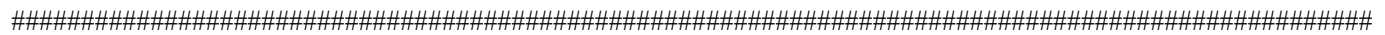

\# You can change variables within this section

$\begin{array}{ll}\mathrm{m}=30 & \text { \# Maximum number of multiple tests to perform } \\ \mathrm{k}=5000 & \text { \# Number of iterations per test } \\ \text { alpha }=0.05 & \end{array}$

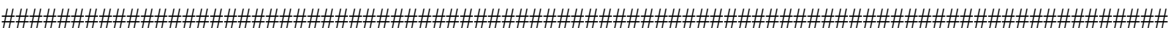

\# Create an array to hold the mean for all the simulations

bonf $=$ np.zeros $(\mathrm{m})$

$\mathrm{bh}=\mathrm{np} \cdot \mathrm{zeros}(\mathrm{m})$

by $=n p \cdot z e r o s(m)$

narum $=n p . z e r o s(m)$

\# Loop structure to perform iterative tests for percent of false positives for $\mathrm{x}$ in range $(\mathrm{m})$ :

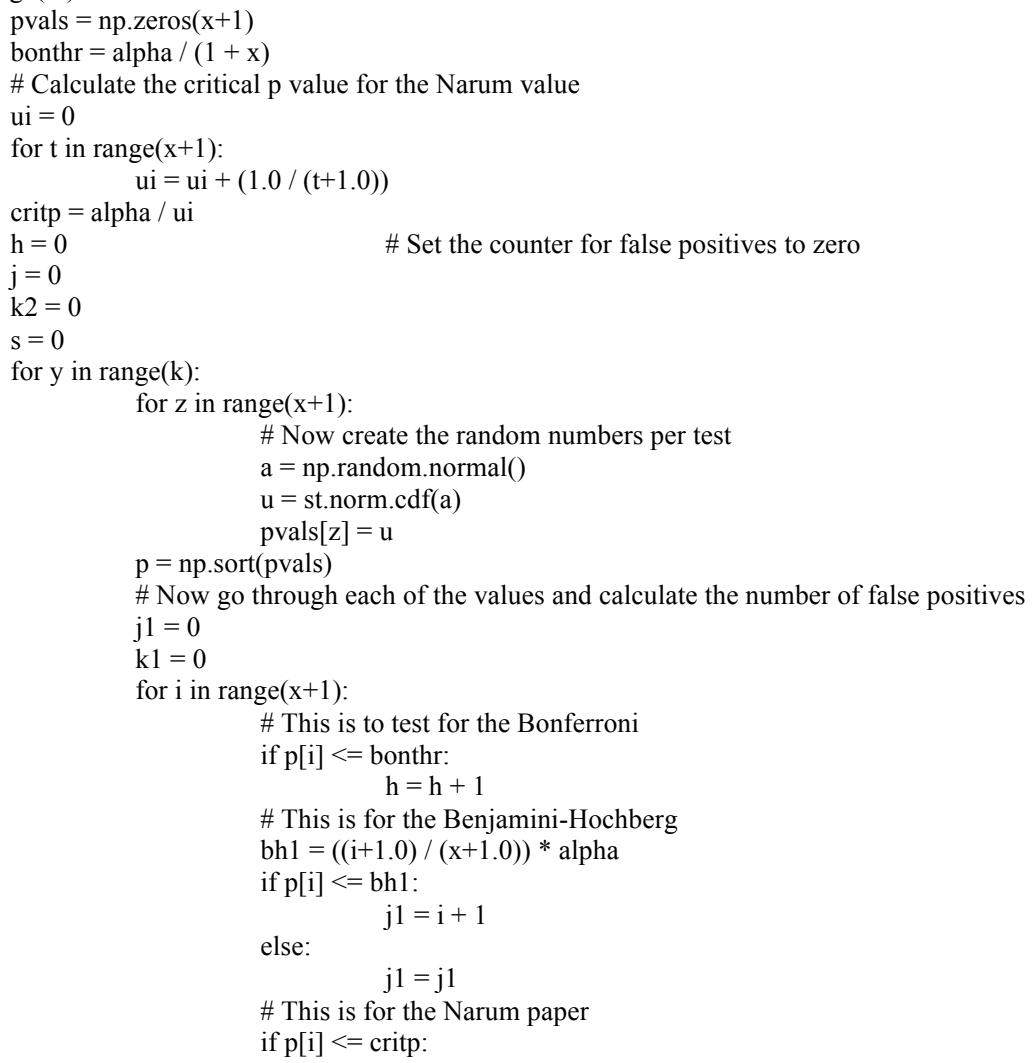


bioRxiv preprint doi: https://doi.org/10.1101/408997; this version posted September 8,2018 . The copyright holder for this preprint (which was not certified by peer review) is the author/funder, who has granted bioRxiv a license to display the preprint in perpetuity. It is made available under aCC-BY 4.0 International license.

$$
\begin{aligned}
& \mathrm{s}=\mathrm{s}+1 \\
& \text { \# This is for the Benjamini-Yekutieli equation } \\
& \text { bh2 }=((\mathrm{i}+1.0) /(\mathrm{x}+1.0)) * \text { critp } \\
& \text { if } \mathrm{p}[\mathrm{i}]<=\mathrm{bh} 2 \text { : } \\
& \mathrm{k} 1=\mathrm{i}+1 \\
& \text { else: } \\
& \mathrm{k} 1=\mathrm{k} 1 \\
& \mathrm{j}=\mathrm{j}+\mathrm{j} 1 \\
& \mathrm{k} 2=\mathrm{k} 2+\mathrm{k} 1 \\
& \text { fwefdr_bh }[\mathrm{x}]=((\mathrm{j} * 1.0) /(\mathrm{k} * 1.0) * 100.0) \\
& \text { fwefdrnarum }[\mathrm{x}]=((\mathrm{s} * 1.0) /(\mathrm{k} * 1.0) * 100.0)
\end{aligned}
$$$$
\text { fwefdr bonf }[\mathrm{x}]=((\mathrm{h} * 1.0) /(\mathrm{k} * 1.0) * 100.0)
$$$$
\text { fwefdr_by }[\mathrm{x}]=((\mathrm{k} 2 * 1.0) /(\mathrm{k} * 1.0) * 100.0)
$$

plt.plot(bonf)

plt.plot(bh)

plt.plot(by)

plt.plot(narum)

plt.show() 